\title{
A new approach to information loss (no) problem for Black Holes
}

\author{
Nikolaos D. Pappas \\ Division of Theoretical Physics, Department of Physics, \\ University of Ioannina, Ioannina GR-45110, Greece
}

\begin{abstract}
The discovery that black holes emit thermal type radiation changed radically our perception of their behavior. Until then, their interior was considered as causally disconnected from the rest of the universe, so any kind of information, that went down the black hole, was believed to remain eternally trapped in it. The emission of the aforementioned radiation means that some amount of information eventually returns to the universe outside the black hole. The question then rises whether it is the whole of this information that goes back to the universe during the black hole evaporation or not. Numerous theories supporting either information preservation or extinction have been developed ever since. A new idea is proposed, based on a deep re-examination of what information is and what are its properties. We postulate that not all kinds of information are of equal importance to nature and, as a result, some of them should be preserved under any conditions, while the rest are allowed to be destroyed, so both preservation and destruction of information is what actually happens during the black hole formation/evaporation process.
\end{abstract}




\section{Introduction}

More than 30 years ago Hawking proved, using a semiclassical approximation, that black holes radiate because of the inevitable creation of pairs of particles due to quantum energy fluctuations at the vicinity of their horizon. Furthermore, he was able to show that this radiation is exactly thermal, that is no subtle or secret correlations exist between the emitted particles [1, 2]. As we know nowadays, black holes actually behave like black bodies of a specific temperature $\mathrm{T}$ given by the equation

$$
T=\frac{c^{3} \hbar}{8 \pi k_{B} G M}
$$

Or, in terms of solar mass $M_{\odot}$,

$$
T=6.2 \times 10^{-8}\left(\frac{M_{\odot}}{M}\right) K
$$

Hawking radiation drew much attention since then as, for the very first time, physicists dealt with a procedure which results from the combination of a purely quantum mechanical process, such as particle creation from vacuum, with the dynamical properties of space-time, that are governed by the laws of General Relativity. Space-time is no longer considered as the passive background where quantum phenomena take place, but, on the contrary, its curvature and the existence of an event horizon are indispensable in order for a black hole to emit particles. Nevertheless, even quantum phenomena must abide by the energy conservation principle. Hence, when Hawking radiation escapes to infinity, we may safely conclude that it will carry energy away from the black hole, which must therefore shrink in mass. As the mass shrinks the surface gravity increases and with it the temperature. This is a self-catalyst process in which the entire mass evaporates away in a finite time.

For astrophysical black holes of mass $M$ this procedure is particularly slow and their lifetime, although finite, is very long, calculated to be of the order

$$
\tau_{B H} \sim\left(\frac{M}{m_{p l}}\right) t_{p l} \sim\left(\frac{M}{M_{\odot}}\right)^{3} 10^{71} \mathrm{sec}
$$

where $m_{p l} \approx 10^{-5} \mathrm{gr}$ is the Planck mass and $t_{p l} \approx 10^{-43} \mathrm{sec}$ is the Planck time. All these mean that the estimated lifetime of a solar mass black hole is $10^{53}$ times larger than the current age of our universe, whose Hubble time is $t_{H}=H_{0}^{-1} \propto 10^{18} \mathrm{sec}$. The duration of the process may seem extremely long for the evaporation of black holes to have any practical impact on us, but one should notice that the lifetime of mini black holes is so much shorter that primordial black holes could reach the end of their lives today. Even more, since we are talking about the very principles of Physics, no Gedankenexperiment can be regarded as extreme enough so as not to be worth considering.

The evaporation, that comes as a result of the aforementioned radiation, posed an unexpected question. Initially, the interior of black holes used to be considered as causally disconnected from the rest of the universe, so any kind of information, that gets inside a black hole, was believed to remain eternally trapped in it. We know that this is not 
the case, as black holes radiate, and, therefore, some amount of information eventually returns back to our universe. The major question, accompanying this observation, is whether, during the formation/evaporation process of a black hole, information is preserved or gets partially destroyed.

Every scientist, that concerns him/herself with this issue, before anything else, always bumps into the question whether information is actually preserved or destroyed. Whatever the answer, the effort to support it gives rise to new questions and challenges. If information is conserved, then one should propose or invent some kind of a mechanism, which ensures this. Bearing in mind that we lose track of some amount of information in every ordinary process, why should black holes preserve it in the first place? If, on the other hand, one accepts the possibility that information can be destroyed, questions about when and why this happens should be addressed. Being it the case, what makes a black hole to abstain from destroying the whole of it and lose even its classical hair? And for the whole matter to get more complicated, unitarity, a key demand of Quantum Theory, appears to be violated in the context of the formation and evaporation of black holes since particles in pure state, that get absorbed by them, end up in mixed state as parts of Hawking radiation.

All these questions outline the celebrated information loss problem, which has been tantalizing physicists for more that three decades. This problem actually consists of two quite different issues. The first one could be described as "loss of history" meaning that two black holes of the same mass, charge and angular momentum radiate exactly the same way, even though they probably have absorbed different objects during their lifetime. Therefore, we lose knowledge about the specific properties of whatever goes down a black hole apart from the three parameters mentioned above. The second one has to do with the apparent non-unitary evolution of particles that black holes seem to evoke, as stated earlier. Here we will only deal with the "loss of history" problem and leave the salvation of unitarity to be the subject of a future work.

There are numerous papers, where theories about possible preservation mechanisms are presented by several scientists, since most of the physicists find the idea of information destruction and the subsequent breakdown of predictability to be unpalatable. The most significant ones have to do with the invention of some mechanism through which an enormous amount of information can either be encoded in Hawking radiation [3, 4, 5, 6, [7, 8] or is forced to remain trapped in the inaccessible interior of a black hole remnant [9]. Both of them, though, are still far from being considered as complete solution to the problem, since they have serious drawbacks. The existence of complicated but subtle correlations in the spectrum of Hawking radiation possible as it may seem, meaning, of course, that the latter is nearly and not exactly thermal, consists a deviation from our present knowledge that also has to be explained. Furthermore, for the equilibrium between ingoing and outgoing information to hold, Hawking radiation should carry a really huge amount of all kinds of information and it is quite hard for one to see how this could be realized by these alleged correlations that, in any case, are assumed to be very feeble (for a convincing presentation of the arguments undermining the validity of such a solution see [10] and for a proposed way out see [11]). As far as black hole remnants are concerned, their existence is even more problematic as their abundance and total 
mass are calculated to be so large, that their gravitational impact on the known universe should have already been detected. Not to mention that only vague assumptions can be made about what kind of mechanism can stop the evaporation and save black holes from extinction by creating an extremely stable and long-lived remnant, that is left behind at the end [12, 13].

Quite a few other theories have been proposed over the years to address the problem, where information comes out massively once the black hole reaches the Planck size when the semiclassical approximation is no longer trustworthy [14], escapes into a babyuniverse [15], is conserved in space-times of $1+1$ dimensions [16, 17], remains trapped inside the infinitely large interior of cornucopions (a variation of remnants) [18, 19, 20, 21], is stored in a topologically disconnected from our Universe region, created inside the black hole due to a topological change process that the horizon undergoes spontaneously, the latter being a fuzzy sphere in the first place [22] etc. Despite any virtues they may have, these theories suffer from very serious defects that make them least viable (see for example [23] for an extensive and thorough presentation and analysis of various theories both mainstream and exotic ones, and also [24]).

Significantly fewer papers appear in the literature to support the possibility of information destruction [23, 25], since the issue is not whether it can get lost for all practical purposes, but if it can be destroyed in a way that is irreversible in principle. Most of the physicists seem reluctant to defend such a prospect, as one would have to answer why and how this could happen and what are the limitations of this procedure. Nevertheless, our present knowledge implies that this is probably the case even if this means that we should alter or expand some of our ideas concerning how nature works.

\section{Classes of information}

Up till now, it must be evident that the information loss paradox cannot be encountered using only well established and understood rules and theories. Our current lack of knowledge about the laws of Quantum Gravity means that every approach is incomplete to some extend as it is doomed to overlook the fact that the particles spend a part of their lives interacting with the singularity since this interaction can only be understood and calculated in the context of exactly these yet ill understood laws. An innovative idea is proposed in this paper, which can be described in a nutshell as "information classification", that could be proven to be a useful tool in the effort to fully understand the nature of black holes.

We postulate that all kinds of information, in general, about a physical system fall into two categories. Let's call them $\Pi 1$ and $\Pi 2$ ( $\Pi$ is the first letter of the greek word that stands for information). П1 class contains the most fundamental information that defines a particle, such as the mass, the electric and magnetic charge and the angular momentum of it. The much larger $\Pi 2$ category includes information about how particles mingle with each other and the properties that rise from their combinations. A book, for example, contains a vast number of П1-info about the aforementioned conserved quantities, of the elementary particles it consists of, and an even larger number of $\Pi 2$-info about how this 
particles unite to form different nucleons, atoms and molecules including also the way all these combine to form letters and words that mean something.

There is a variety of well known conservation laws that impose the preservation of П1-info by any physical system. Black holes satisfy this requirement by emitting all kinds of particles that carry away exactly this type of information. It should be pointed out here that whether information about leptonic and baryonic numbers or any fermionic degrees of freedom, in general, should be considered as $\Pi 1$ type is an open question. Although we know that black holes have no well-defined leptonic or baryonic number [26, 27, 28, we cannot tell yet if they behave in a way that results to the conservation of fermionic quantum numbers or some combination of them. As for the $\Pi 2$ category, there are no such laws to prevent this information from extinction and, consequently, different processes destroy different amounts of it. Actually, we conjecture that the various kinds of П2-info resist their destruction to different degrees. In general, more violent procedures destroy more of them, but not in a proportional way. For instance, going back to the book example, tearing it apart leads to some П2-info loss, like the meaning of the sentences and the words written in it. By burning it, we destroy much more П2-info, since now words and letters disappear and no paper or ink survives, but the atoms, it was made of, are still there. Because all usual phenomena are confined to a low energy scale, not even all П2-info gets destroyed during them. This means that in every day life we get to observe loss only of some part of the total $\Pi 2$ category practically in every process, which leads to an increase of the total entropy, as dictated by the second law of thermodynamics and no paradoxes occur. However, the case of black holes is somewhat different in that, being the most extreme objects in the universe, the matter, they absorb, undergoes impacts of arbitrarily large violence, so they destroy all П2-info ruthlessly and, as a result, their entropy adds up to enormous values. Meanwhile П1-info remains intact even in this case.

In a few words, we argue that the concept of information loss as the physical basis of the second law of thermodynamics [29] should be completed with the limitation that this loss can only concern П2-info, no matter how easy or difficult it is to be destroyed. Furthermore, black holes are postulated to be the most efficient $\Pi 2$-info destroyers in the Universe and no such info can survive after having crossed the horizon. Rephrasing a well known aphorism by R. Price [30], "any $\Pi 2$ type piece of information that could be destroyed by a black hole, will be destroyed".

\section{Information paradox resolution}

Taking under consideration the idea of information classification, we gain a more incisive perception of the behavior of black holes, which has significant advantages and almost no flaws. To be more specific there are six arguments in favor of the new idea.

First,information, as far as the subset $\Pi 1$ of fundamental importance is concerned, is never lost and, therefore, no paradox rises at this level.

Second, because information does get destroyed by the black hole, even if it can only be of $\Pi 2$ category, the absorption and incorporation of matter by the black hole is a thermodynamically favored procedure, as expected by the fact that this is what always 
happens in reality.

Third, since black holes interact with the rest of the universe via their horizon, the rate they absorb matter and, consequently, destroy the information contained in it, must be proportional to their surface A. This observation provides us with a possible explanation of why black hole entropy is directly analogous to their surface, as explicitly shown by the famous Bekenstein - Hawking formula

$$
S=\frac{A}{4} \quad \text { with } \quad c=G=\hbar=1
$$

and not to their volume, as could one instinctively assume accounting black holes to be some sort of ordinary thermodynamical systems.

Fourth, everything we know so far about the behavior of black holes and the laws governing it, like the Generalized Second Law, remain intact and valid since no revision of them is necessary in the context of our theory.

Fifth, it provides us with some mechanism capable of explaining why black hole entropy generally takes enormous values, as we can ascribe it to the complete and irreversible destruction of all П2-info.

Sixth, the fact that less information contributes to the creation of the entropy of black holes, since they destroy only П2-info, even though П2 class constitutes the greater part of the total information load of incoming matter, can help us deal with the problematic current estimation that the entropy of ordinary black holes is almost equally large as the entropy of the Universe. More specifically, the latter is approximately equal to the number of relativistic particles whose number, within a Hubble radius, is calculated to be $S_{U}=10^{88}$. On the other hand, black hole entropy measured in Planck units is equal to the $1 / 4$ of their surface and converting this quantity into astrophysical units we find the Bekenstein - Hawking entropy to have the huge value

$$
S_{B H} \sim 10^{90}\left(\frac{M}{10^{6} M_{\odot}}\right)^{2}
$$

This equation means that a single million solar mass black hole has more entropy than the whole known universe. At the same time General Relativity certainly allows for the existence of such supermassive objects and astronomical observations imply that they must exist in the centre of almost every galaxy, so another paradox rises. A possible way out is that the idea of information classification also means that the way black hole entropy is calculated should be reconsidered on the basis of $\Pi 1$ and $\Pi 2$ information classes in order to express the fact that only $\Pi 2$-info gets destroyed. The refined calculation should result to an entropy value several orders of magnitude smaller than the one mentioned above, resolving the new paradox.

Finally, information classification theory is quite appealing in that, while predicting some kind of information conservation, avoids any assumptions about remnants or exotic encoding in the spectrum of Hawking radiation or other even more radical but least possible alternatives. Its simplicity should be seen as an extra advantage of it, if one is to trust Occam and his famous razor 1 .

\footnotetext{
${ }^{1}$ Occam's razor: philosophical argument stating that among all theories, capable of explaining a
} 
Of course, it is still an open question what actually happens to unitarity, demanded by Quantum Theory, during the formation/evaporation process of black holes for which the information classification has nothing new to propose. As mentioned before, our idea only deals with the "loss of history" part of the paradox and, in our opinion, seems to work very well indeed.

\section{Conclusions}

Information loss paradox is a very interesting and still open issue that indicates the limits of the $20^{t h}$ century physics and waits for its solution to be found, hopefully, in the $21^{\text {st }}$ century. Several efforts have been made so far to resolve it, but all of them have such serious drawbacks, that hardly any can be considered as a good start for a definitive answer. In this paper, a differentiation in the way we perceive the notion of information is proposed, based on the assumption that not all kinds of information are equally important to nature. We argue that some of them are of fundamental and others of secondary importance, that are characterized as $\Pi 1$ and $\Pi 2$ respectively, on the basis that the first ones are protected by a series of conservation laws against destruction, while the latter ones are allowed to be destroyed with different degrees of ease. Postulating that black holes radiate away all $\Pi 1$ information through Hawking radiation and, at the same time, they destroy all of the $\Pi 2$ one, we manage to avoid any paradoxes, while their behavior remains compatible with the second law of thermodynamics. In this way, one can claim that the information loss paradox, as far as the "loss of history" is concerned, is resolved, at least in principle.

phenomenon, the one with the simplest conjectures is most probably the correct one. 


\section{References}

[1] S. W. Hawking, Commun. Math. Phys. 43, 199 (1975).

[2] J. B. Hartle and S. W. Hawking, Phys. Rev. D 13, 2188 (1976).

[3] S. W. Hawking, Phys. Rev. D 72, 084013 (2005); hep-th/050717.

[4] J. D. Bekenstein and V. F. Mukhanov, Phys. Lett. B 360, 7 (1995).

[5] C. R. Stephens, G. 't. Hooft and B. F. Whiting, Class. Quant. Grav. 11, 621 (1994); gr-qc/9310006.

[6] S. Hod, Phys. Lett. A 299, 144 (2002); gr-qc/0012076.

[7] V. F. Mukhanov, Pis'ma. Zh. Eksp. Teor. Fiz. 44, 50 (1986).

[8] T. Vachaspati, D. Stojkovic, L. M. Krauss, Phys. Rev. D 76, 024005 (2007); gr-qc/0609024.

[9] Y. Aharonov, A. Casher and S. Nussinov, Phys. Lett. B 191, 51 (1987).

[10] S. D. Mathur, Class. Quant. Grav. 26, 224001 (2009); hep-th/0909.1038.

[11] T. Vachaspati, D. Stojkovic, Phys. Lett. B 663, 107-110 (2008); gr-qc/0701096.

[12] J. D. Bekenstein, Phys. Rev. D 49, 1912 (1994).

[13] L. Susskind, hep-th/9501106.

[14] D. N. Page, Phys. Rev. Lett. 71, 3743 (1993); hep-th/9306083.

[15] V. Frolov, S. B. Giddings, A. Strominger, P. C. Argyres et al., astro-ph/9412046.

[16] A. Ashtekar, V. Taveras and M. Varadarajan, Phys. Rev. Lett. 100, 211302 (2008); gr-qc/0801.1811.

[17] A. Peet, L. Susskind and L. Thorlacius, Phys. Rev. D 46, 3435 (1992); hep-th/9205114.

[18] T. Banks, M. O'Loughlin and A. Strominger, Phys. Rev. D 47, 4476 (1993); hep-th/9211030.

[19] T. Banks, Nucl. Phys. Proc. Suppl. 41, 21 (1995); hep-th/9412131.

[20] S. B. Giddings, Phys. Rev. D 46, 1347 (1992).

[21] T. Banks, A. Dabholkar, M. R. Douglas and M. O'Loughlin, Phys. Rev. D 45, 3607 (1992).

[22] C. A. S. Silva, Phys. Lett. B 677, 318-321 (2009); gr-qc/0812.3171.

[23] J. Preskil, hep-th/9209058. 
[24] S. B. Giddings, hep-th/9209113.

[25] S. W. Hawking, Phys. Rev. D 14, 2460 (1976).

[26] J. B. Hartle, Phys. Rev. D 1, 394 (1970); Phys. Rev. D 3, 2938 (1971).

[27] C. Teitelboim, Lett. Nuovo Cim. 3, 326 (1972); Lett. Nuovo Cim. 3, 397 (1972); Phys. Rev. D 5, 2941 (1972).

[28] J. D. Bekenstein, Phys. Rev. Lett. 28, 452 (1972); Phys. Rev. D 5, 1239 (1972); Phys. Rev. D 5, 2403 (1972).

[29] T. L. Duncan and J. S. Semura, Foundations of Physics 37, 1767 (2007).

[30] R. H. Price, Phys. Rev. D 5, 2419 (1972); Phys. Rev. D 5, 2439 (1972). 